\title{
Epidemiological, otolaryngological, olfactory and gustatory outcomes according to the severity of COVID-19: a study of 2579 patients
}

\author{
Jerome R. Lechien ${ }^{1,2,3,4}$ (D) Carlos M. Chiesa-Estomba ${ }^{1,5} \cdot$ Luigi A. Vaira $^{6}$ - Giacomo De Riu ${ }^{6}$. Giovanni Cammaroto ${ }^{1,7}$.

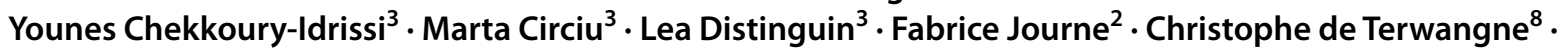 \\ Shahram Machayekhi ${ }^{8} \cdot$ Maria R. Barillari $^{1,9} \cdot$ Christian Calvo-Henriquez $^{10} \cdot$ Stéphane Hans $^{3} \cdot$ Sven Saussez ${ }^{1,2,4}$
}

Received: 22 November 2020 / Accepted: 2 December 2020 / Published online: 16 January 2021

(c) The Author(s), under exclusive licence to Springer-Verlag GmbH, DE part of Springer Nature 2021, corrected publication 2021

\begin{abstract}
Objective To investigate prevalence and epidemiological and clinical factors associated with olfactory dysfunction (OD) and gustatory dysfunction (GD) in COVID-19 patients according to the disease severity.

Study design Cross-sectional study.

Methods A total of 2579 patients with a positive diagnosis of COVID-19 were identified between March 22 and June 3, 2020 from 18 European hospitals. Epidemiological and clinical data were extracted. Otolaryngological symptoms, including OD and GD, were collected through patient-reported outcome questionnaire and Sniffin'Sticks tests were carried out in a subset of patients.

Results A total of 2579 patients were included, including 2166 mild (84.0\%), 144 moderate (5.6\%) and 269 severe-to-critical $(10.4 \%)$ patients. Mild patients presented an otolaryngological picture of the disease with OD, GD, nasal obstruction, rhinorrhea and sore throat as the most prevalent symptoms. The prevalence of subjective OD and GD was 73.7 and 46.8\%, and decreases with the severity of the disease. Females had higher prevalence of subjective OD and GD compared with males. Diabetes was associated with a higher risk to develop GD. Among the subset of patients who benefited from psychophysical olfactory evaluations, there were 75 anosmic, 43 hyposmic and 113 normosmic patients. The prevalence of anosmia significantly decreased with the severity of the disease. Anosmia or hyposmia were not associated with any nasal disorder, according to SNOT-22.

Conclusion OD and GD are more prevalent in patients with mild COVID-19 compared with individuals with moderate, severe or critical diseases. Females might have a higher risk of developing OD and GD compared with males.
\end{abstract}

Keywords COVID-19 Coronavirus · SARS-CoV-2 Anosmia $\cdot$ Hyposmia $\cdot$ Olfactory $\cdot$ Smell $\cdot$ Outcome Epidemiological $\cdot$ Clinical

Jerome R. Lechien and Carlos M. Chiesa-Estomba have equally contributed to this work and should be regarded as joint first authors.

Stéphane Hans and Sven Saussez have equally contributed to the work supervision and are co-senior authors.

Jerome R. Lechien

Jerome.Lechien@umons.ac.be

Extended author information available on the last page of the article

\section{Introduction}

As of November 22, 2020, there were 57,882,183 confirmed cases of coronavirus disease 2019 (COVID-19) worldwide, including $1,377,395$ deaths. While the pandemic seems to be under control in Europe and Asia, the number of cases in North and South America is still high [1]. The clinical picture of the infection includes fever, cough and dyspnea but recent studies reported a high prevalence of otolaryngological symptoms, especially in mild patients [2], including rhinorrhea, nasal obstruction, throat pain and dysphonia $[2,3]$. A special attention has been paid to olfactory (OD) and gustatory dysfunctions (GD) because they may concern a significant number of patients $[2,4,5]$. The majority of 
studies investigating OD or GD have included patients with mild form of the disease, with, consequently, few considerations of patients with moderate, severe or critical diseases. To date, a few data are available in the literature about the prevalence of OD and GD according to the severity of the disease and little is known about the conditions associated with the development of OD and GD.

The aim of this study is to investigate the prevalence and the associated conditions of GD and OD development in COVID-19 patients according to the severity of the disease.

\section{Methods}

\section{Ethical statements}

The study was approved by five European Institutional Review Boards (blinded for review). An electronic informed consent was obtained.

\section{Participants and definition}

Patients were included from 18 European hospitals between March 22nd and June 3rd, 2020. The COVID-19 diagnosis was based on nasal swabs and virus identification with reverse transcriptase polymerase chain reaction (RT-PCR). Some patients benefited from serology (IgM and $\operatorname{IgG})$. The patients had nasal swabs after presenting COVID-19-related symptoms. They were categorized into mild, moderate, severe and critical COVID-19 patients according to the COVID-19 Disease Severity Scoring of the World Health Organization [6]. Mild patients consisted of home-managed patients without evidence of viral pneumonia or hypoxia. Moderate COVID-19 patients were defined as individuals with clinical findings of pneumonia (e.g., fever, cough, dyspnea, or tachypnea) but no sign of severe pneumonia (including $\mathrm{SpO} 2 \geq 90 \%$ on room air). The disease was categorized as severe when patients showed clinical findings of pneumonia associated with one of the following features: respiratory rate $>30$ breaths/min; severe respiratory distress; or $\mathrm{SpO} 2<90 \%$ on room air. Subjects with critical disease had acute respiratory distress syndrome, sepsis or septic shock. These patients were admitted into intensive care units (ICU).

\section{Demographic, epidemiological and clinical outcomes}

The following demographic and epidemiological data were collected with a standardized online questionnaire: gender; age; ethnicity; smoking and comorbidities (i.e., heart, respiratory, kidney, liver, neurological, autoimmune diseases, diabetes, hypertension and gastroduodenal disorders). Mildto-moderate patients fulfilled themselves the questionnaire, while physicians completed the questionnaire after anamnesis or on medical record for the hospitalized patients who were not able to electronically complete the evaluations.

From a clinical standpoint, general symptoms were assessed as present or absent. Otolaryngological symptoms were rated with a 5-point scale ranging from 0 (absent) to 4 (very severe symptoms). Both subjective olfactory and gustatory functions were assessed through items of the smell and taste component of the National Health and Nutrition Examination Survey [7].

\section{Psychophysical evaluations}

Sniffin'Sticks tests (Medisense, Groningen, Netherlands) were used to assess the psychophysical evaluations in a subset of patients who came from three hospitals (blinded for review). The Sniffin'Sticks test is a validated psychophysical olfactory test using 16 smell pens. Each pen was presented to individual who had to choose the adequate smell between 4 given options [8]. The final score ranges from 0 (no olfaction) to 16 (perfect olfaction). Normosmia consists of score between 12 and 16; hyposmia is defined by a score ranging from 9 to 11 and anosmia is defined with a score $<9$ [8]. The evaluations were made within the 2 weeks of the onset of the olfactory disease in mild and moderate patients and 3-4 weeks for severe or critical patients who were in ICU. This subgroup of patients filled in the French version of the sinonasal outcome tool-22 (SNOT-22) [9] simultaneously with Sniffin'Sticks tests and the subjective evaluations. SNOT-22 was used to assess the presence of nasal complaints and the potential relationship between nasal obstruction/rhinorrhea and OD.

\section{Statistical analyses}

Statistical analyses were performed using the Statistical Package for the Social Sciences for Windows version 22.0 (IBM Corp, Armonk, NY, USA). According to the type of outcomes, the following tests were used to compare severity groups: Kruskal-Wallis, Chi-square and Mann-Whitney $U$ test. Multivariate analysis was used to study the associations between outcomes. Because there were few critical COVID19 patients, we considered severe and critical forms in a single group for the analyses.

\section{Results}

A total of 2579 patients were included. There were 2166 mild (84.0\%), 144 moderate (5.6\%), and 269 severe-to-critical $(10.4 \%)$ patients, respectively. The flow chart of the study is available in Fig. 1. The demographic, epidemiological and clinical outcomes of patients are available in Table 1 . The 


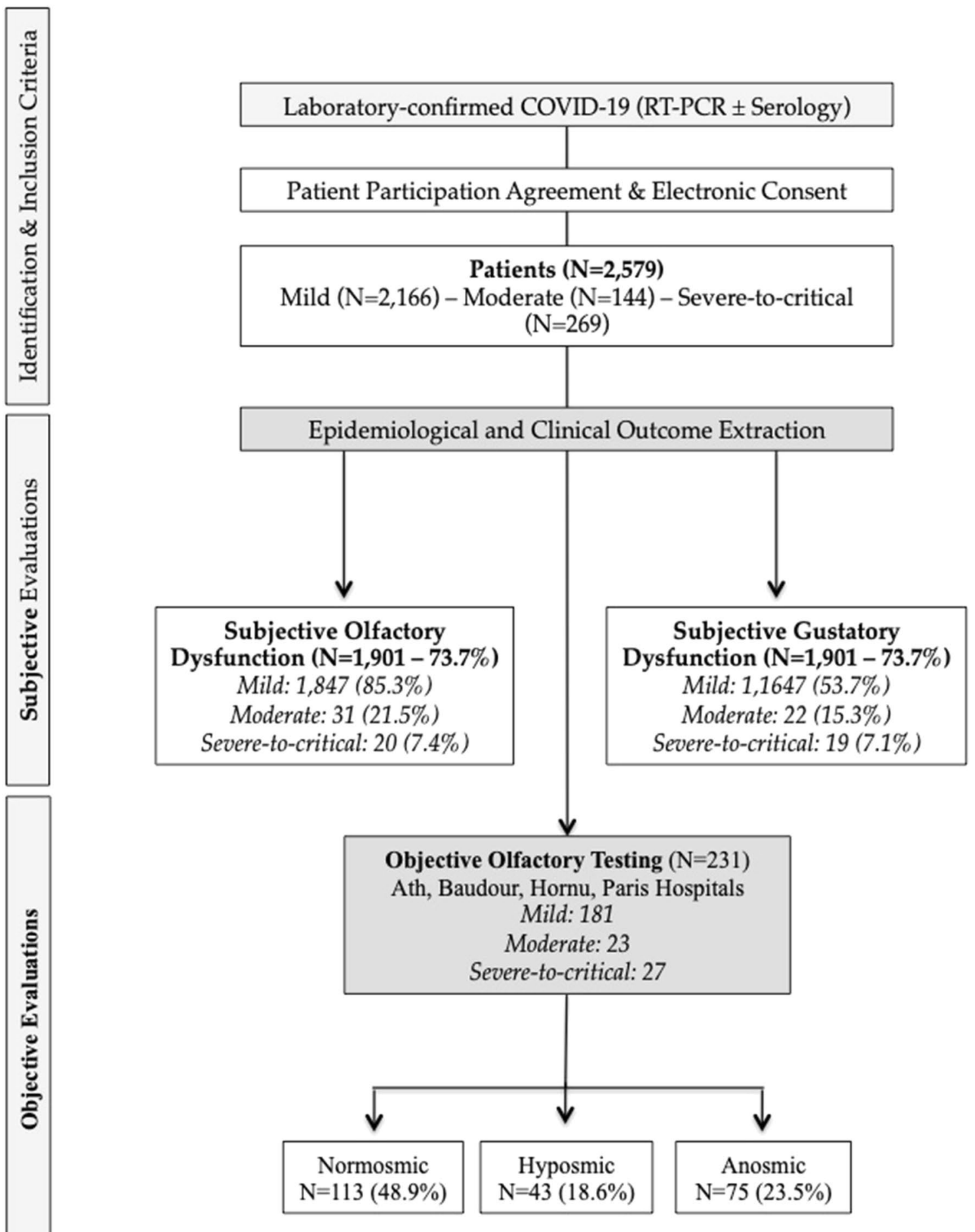

Fig. 1 Flow chart. COVID-19 coronavirus disease 2019; $R T-P C R$ reverse transcriptase polymerase chain reaction

following ethnicities were represented: Caucasia (89.1\%), South America (5.1\%), North Africa (2.0\%), Black Africa (1.2\%) or other $(2.6 \%)$. There were 1630 women $(63.2 \%)$. The mean age was $44.4 \pm 16.7$ years old. The severe-tocritical patients were older $(71.7 \pm 13.7)$ than the moderate
$(63.9 \pm 18.5)$ and mild $(39.6 \pm 12.0 ; p=0.001)$ patients. The female/male ratio of mild, moderate and severe-to-critical patients was $2 / 1,1 / 1$ and $1.1 / 1.6$, respectively $(p<0.05)$.

The most prevalent comorbidities were hypertension (16.0\%), diabetes $(5.9 \%)$, gastroesophageal disorders (i.e., 
Table 1 Patient characteristics

\begin{tabular}{ll}
\hline Characteristics & Patients $(N=2579)$ \\
\hline Age (y-Mean; SD) & $44.4 \pm 16.7$ \\
Gender (F/M) & $1630 / 949$ \\
Current smoker & $362(14.0)$ \\
History of seasonal allergy & $460(17.8)$ \\
Comorbidities & \\
Diabetes & $152(5.9)$ \\
Hypertension & $412(16.0)$ \\
Renal insufisiency & $59(2.3)$ \\
Liver insufisiency & $38(1.5)$ \\
Respiratory insufisiency & $65(2.5)$ \\
Gastroesophageal disorder & $128(5.0)$ \\
Heart disorder & $126(4.9)$ \\
Neurological disorder & $101(3.9)$ \\
Autoimmun disorder & $25(1.0)$ \\
General symptoms $(N-\%)$ & \\
Headache & $1542(59.8)$ \\
Cough & $1423(55.2)$ \\
Myalgia & $1379(53.5)$ \\
Dyspnea & $1165(45.2)$ \\
Fever $(>38 C)$ & $1087(42.1)$ \\
Anorexia & $1047(40.6)$ \\
Arthralgia & $859(39.5)^{*}$ \\
Diarrhea & $799(31.0)$ \\
Asthenia, malaise or confusion & $764(29.6)$ \\
Chest pain & $258(19.2)^{*}$ \\
Abdominal pain & $462(17.9)$ \\
Nausea, vomiting & $451(17.5)$ \\
Sticky mucus/phlegm & $336(13.0)$ \\
\hline & \\
\hline &
\end{tabular}

$F / M$ female/male; $N$ number; $S D$ standard deviation; $y$ year(s)

reflux and gastric ulcer) (5.0\%) and heart disorders (4.9\%). Moderate and severe-to-critical COVID-19 patients had higher prevalence of hypertension (60.5 versus 7.9$)$, diabetes (27.6 versus 1.9), gastroesophageal disorders ( 8.52 versus $4.0)$, renal (12.1 versus 0.5$)$, respiratory (14.0 versus 0.5$)$, heart (16.0 versus 1.8$)$, liver (4.4 versus 0.8$)$ and neurological disorders (13.7 versus 0.6$)$ than mild patients $(p<0.05)$.

\section{General and otolaryngological outcomes}

The prevalence of general symptoms is reported in Table 1 . The most prevalent general symptoms were headache, cough and myalgia. The prevalence and severity of otolaryngological symptoms are reported in Table 2. Nasal obstruction, rhinorrhea and sore throat were the most prevalent symptoms associated with COVID-19. According to the NHNES questions, 1901 (73.7\%) and 1205 (46.8\%) patients complained from subjective OD and GD. The distribution of the most prevalent general and otolaryngological symptoms according to the severity groups is available in Fig. 2. Mild COVID-19 patients had more frequently otolaryngological symptoms compared with moderate and severe-to-critical patients.

\section{Gustatory dysfunction}

The subjective GD, which was defined by impairment of the following four taste modalities: salty, sweet, bitter and sour [6], was reported in 53.7\% $(N=1164), 15.3 \%(N=22)$ and $7.1 \%(N=19)$ of mild, moderate and severe-to-critical COVID-19 patients, respectively (Table 3 ). The prevalence of GD was significantly higher in mild patients compared with moderate and severe-to-critical patients $(p=0.001)$. Aroma dysfunction (AD), which was defined as impairment of perception of tasting flavors in food and/or drinks that are not salty, sweet, bitter or sour (like chocolate, banana and fish), was evaluated in 2105 patients. Among them, aroma perception was reduced in 1116 patients $(53.0 \%)$, loss in 330 subjects $(15.7 \%)$ and distorted in 330 patients (15.7\%). The overall prevalence of AD was $84.4 \%$. AD concerned $80.7 \%$ of mild patients, while moderate and severe-to-critical COVID-19 patients had AD (distortion, reduction or loss) in 35.3 and $17.2 \%$ of cases, respectively (Table 3 ). The
Table 2 Otolaryngological symptom severity of COVID-19 patients

\begin{tabular}{llllllr}
\hline & & & \multicolumn{2}{l}{ Otolaryngological Symptom Severity } & \\
\cline { 4 - 6 } \cline { 4 - 5 } & Prevalence & Absent (0) & Mild (1) & Moderate (2) & Severe (3) & Very severe (4) \\
\hline Nasal obstruction & 53.7 & $1193(46.3)$ & $517(20.1)$ & $388(15.0)$ & $307(11.9)$ & $174(6.7)$ \\
Rhinorrhea & 49.5 & $1302(50.5)$ & $653(25.3)$ & $363(14.1)$ & $193(7.5)$ & $68(2.6)$ \\
Sore throat & 41.9 & $1498(58.1)$ & $512(19.9)$ & $298(11.6)$ & $175(6.8)$ & $96(3.7)$ \\
Postnasal drip & 39.4 & $1562(60.6)$ & $481(18.7)$ & $271(10.5)$ & $161(6.2)$ & $104(4.0)$ \\
Face pain/heaviness & 35.3 & $1669(64.7)$ & $324(12.6)$ & $236(9.2)$ & $202(7.8)$ & $148(5.7)$ \\
Ear pain & 21.4 & $2028(78.6)$ & $287(11.1)$ & $138(5.4)$ & $89(3.5)$ & $37(1.4)$ \\
Dysphonia & $27.8 *$ & $619(72.2)$ & $144(16.8)$ & $54(6.3)$ & $29(3.4)$ & $11(1.3)$ \\
Dysphagia & 17.0 & $2141(83.0)$ & $227(8.8)$ & $123(4.8)$ & $56(2.2)$ & $32(1.2)$ \\
\hline
\end{tabular}

*Dysphonia was not assessed on all patients 


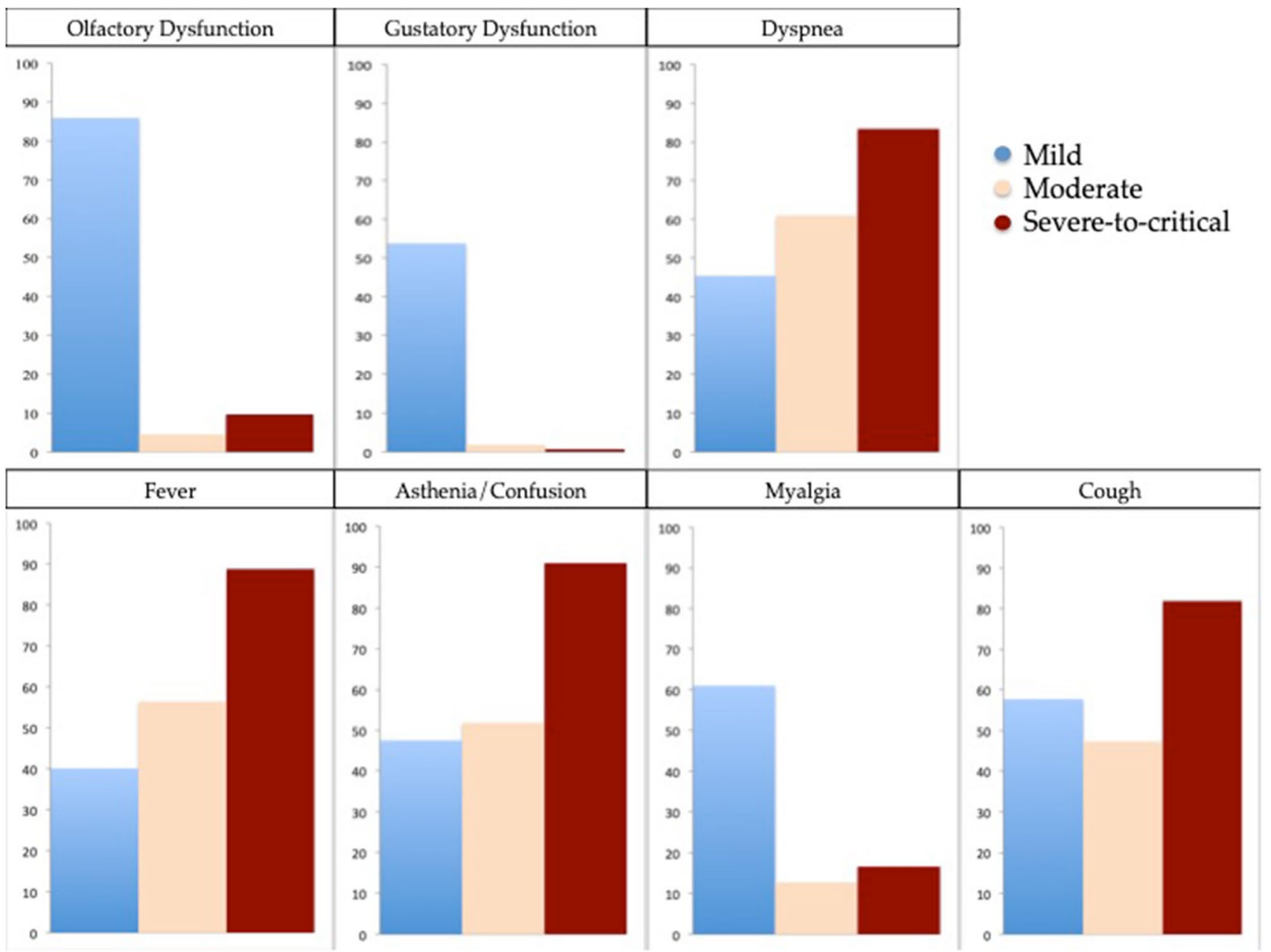

Fig. 2 Distribution of the most prevalent general and otolaryngological symptoms according to the severity

prevalence of $\mathrm{AD}$ was significantly higher in mild patients compared to moderate-to-critical $(p=0.001)$.

The development of subjective GD was positively associated with the presence of diabetes $(p=0.001)$. Diabetes was also associated with the development of $\operatorname{AD}(p=0.013)$. Patients with hypertension had a lower proportion of AD (47.0\%) compared with those without hypertension (73.5\%; $p=0.003)$. Smokers reported more frequently AD (73.3\%) compared with non-smoking COVID-19 patients (67.3\%; $p=0.002$ ). Among patients with AD, non-smokers more frequently reported reduction of aroma perception compared with smokers who reported total loss or distortion of aroma perception.

\section{Olfactory dysfunctions}

Among the patients with OD 1545 (59.9\%) and 356 (13.8\%) reported that the OD consisted of partial or total loss of smell. The prevalence of subjective OD in mild, moderate and severe-to-critical patients was $85.3 \%(N=1847), 21.5 \%$
$(N=31)$ and $7.4 \%(N=20)$, respectively $(p=0.001)$. Cacosmia and phantosmia were reported in 63.6\% $(N=1427 / 2242)$ and $14.1 \%(N=316 / 2242)$ of cases. Irrespective of disease severity, the subjective OD developed before (14.6\%), during $(25.4 \%)$ or after $(55.0 \%)$ the other symptoms. The mean duration time of OD was $11.5 \pm 5.7$ days.

Among the 2579 patients, 231 patients benefited from Sniffin'Sticks tests, including 181 mild, 23 moderate and 27 severe-to-critical individuals. The median time between the onset of OD and the realization of psychophysical evaluation was 18 days. The mean Sniffin'Stick test was 10.5 \pm 3.7 . There were 75 anosmic, 43 hyposmic and 113 normosmic patients, corresponding to a prevalence of psychophysical OD of $51.0 \%$ (Table 3). Precisely, Sniffin'Stick test was $9.9 \pm 3.7,11.3 \pm 4.1$ and $13.1 \pm 2.0$ in mild, moderate and severe groups ( $p=0.001$; Fig. 3$)$. The numbers of patients with hyposmia and anosmia in mild group were 36 (19.9\%) and $63(34.8 \%)$.

There were $4(17.4 \%)$ and $9(39.1 \%)$ hyposmic and anosmic individuals in moderate patient group. In severe group, 
Table 3 General, olfactory, gustatory and aroma outcomes of COVID-19 patients

\begin{tabular}{|c|c|}
\hline Subjective OD and GD & Number $(\%)$ \\
\hline Self-reported GD & $N=2579$ \\
\hline All patients & $1205(46.8)$ \\
\hline \multicolumn{2}{|l|}{ Subgroups } \\
\hline Mild & $1164(53.7)$ \\
\hline Moderate & $22(15.3)$ \\
\hline Severe-to-critical & $19(7.1)$ \\
\hline Self-reported aroma sense dysfunction & $N=2105$ \\
\hline All patients & $1776(84.4)$ \\
\hline Total loss of aroma perception sense & $330(15.7)$ \\
\hline Partial loss of aroma & $1116(53.0)$ \\
\hline Distortion & $330(15.7)$ \\
\hline No problem & $329(15.6)$ \\
\hline \multicolumn{2}{|l|}{ Subgroups } \\
\hline Mild & $1753(80.7)$ \\
\hline Moderate & $18(35.3)$ \\
\hline Severe-to-critical & $5(17.2)^{*}$ \\
\hline Self-reported OD & $N=2579$ \\
\hline All patients & $1901(73.7)$ \\
\hline Partial loss of smell & $1545(59.9)$ \\
\hline Total loss of smell & $356(13.8)$ \\
\hline Cacosmia & $1427(63.6)$ \\
\hline Phantosmia & $316(14.1)$ \\
\hline \multicolumn{2}{|l|}{ Subgroups } \\
\hline Mild & $1847(85.3)$ \\
\hline Moderate & $31(21.5)$ \\
\hline Severe-to-critical & $20(7.4)$ \\
\hline Onset of Smell Dysfunction & $N=1955$ \\
\hline Before the other symptoms & $285(14.6)$ \\
\hline Concomittant with other symptoms & $497(25.4)$ \\
\hline After the other symptoms & $1075(55.0)$ \\
\hline Did not remember/Missing data & $98(5.0)$ \\
\hline Mean duration (Mean, SD, days) & $11.5 \pm 5.7$ \\
\hline Psychophysical olfactory tests & $N=231$ \\
\hline Normosmic & $113(48.9)$ \\
\hline Hyposmic & $43(18.6)$ \\
\hline Anosmic & $75(32.5)$ \\
\hline
\end{tabular}

$G D$ gustatory dysfunction; $O D$ olfactory dysfunction; $S D$ standard deviation

there were $3(11.1 \%)$ hyposmic and $3(11.1 \%)$ anosmic patients. The prevalence of psychophysical OD in mild, moderate, and severe-to-critical COVID-19 patients was $54.7,56.5$ and $38 \%$, respectively. The proportion of anosmic and hyposmic patients was significantly higher in mild and moderate patients compared with severe-to-critical patients $(p=0.001)$.

The mean SNOT-22 at the time of the olfactory assessment was $33.5 \pm 20.7$. Mild, moderate and

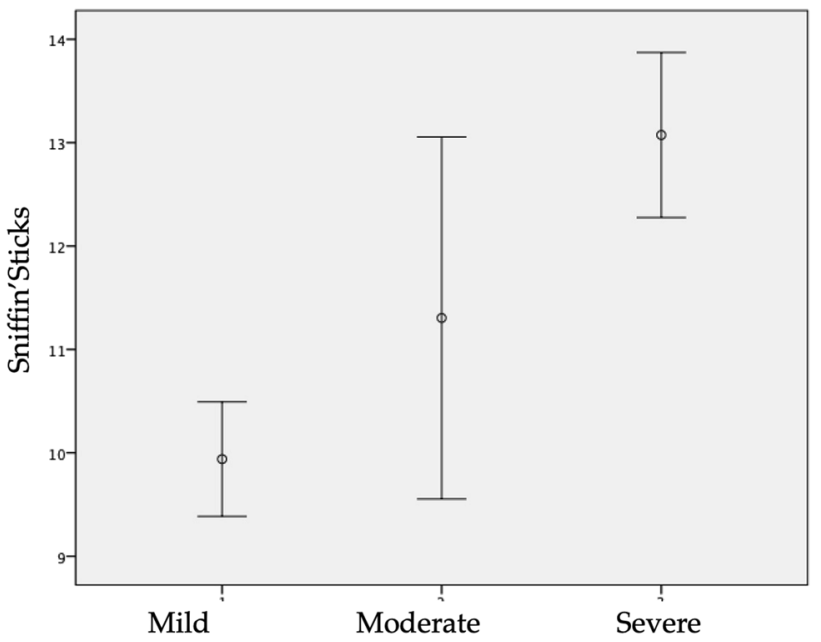

Fig. 3 Mean and standard deviation of Sniffin'Stick test values according to the severity group

severe-to-critical patients had SNOT-22 mean values of $32.9 \pm 20.4,42.6 \pm 20.9$ and $29.4 \pm 21.1$, respectively.

\section{Associations between OD, GD and epidemiological outcomes}

Patients with hypertension had a low proportion of OD than those without hypertension ( $74.9 \%$ versus $88 \% ; p=0.001$ ). Females had higher prevalence of subjective total loss of smell compared with males ( 74.2 versus $65.3 \% ; p=0.001$ ). However, males had a higher proportion of partial loss of smell compared with females (19.2 versus $13.7 \% ; p=0.001)$. Cacosmia was more prevalent in females compared with males $(67.9$ versus $61.7 \%$; $p=0.018)$. Findings were similar for GD (58.4 versus $53.4 \%$; $p=0.038$ ). Moreover, 18.7 and $9.7 \%$ of females and males reported total loss of aroma sense, respectively $(p=0.001)$. Note that aroma perception was distorted in 15.7 and $15.6 \%$ of females and males; while, $11.3 \%$ females and $18.1 \%$ males did not report AD $(p=0.001)$.

Considering patients with psychophysical olfactory testing, we found significant positive associations between the score of Sniffin'Sticks tests and the patient age $\left(r_{\mathrm{s}}=0.246\right.$; $p<0.001)$; fever severity $\left(r_{\mathrm{s}}=0.210 ; p=0.004\right)$, cough $\left(r_{\mathrm{s}}=0.212 ; p=0.003\right)$, dyspnea $\left(r_{\mathrm{s}}=0.243 ; p=0.001\right)$ and asthenia $\left(r_{\mathrm{s}}=0.225 ; p=0.002\right)$; all of these symptoms being more prevalent in patients with moderate and severeto-critical COVID-19 forms. There was a statistical trend for females to have a lower Sniffin' Sticks test result compared with males $(9.7 \pm 4.1$ versus $10.8 \pm 3.6 ; p=0.05)$. The subjective perception of loss of smell was positively associated with the results of the psychophysical evaluations $(p<0.001)$. In other words, patients who reported OD had a higher probability to be anosmic or hyposmic at the 
psychophysical tests. There was a significant association between Sniffin's Sticks tests and self-reported AD. Anosmic and hyposmic patients reported more frequently $\mathrm{AD}$ $(p<0.001)$. There was no significant association between Sniffin'sticks test results and SNOT-22 items and total score.

\section{Discussion}

The recent study of home-managed COVID-19 patients resulted in the identification of an otolaryngological picture of the disease, which could particularly concern mild COVID-19 forms [10, 11]. Among the common otolaryngological symptoms, olfactory and gustatory dysfunctions were reported in many studies conducted around the world [12-14] and are currently considered as prognostic factor for lower severity of COVID-19 [15]. However, to date, a few studies have investigated the prevalence of sense disorders regarding the severity of the infection, as defined by WHO criteria.

In this large cross-sectional study, we observed different clinical pictures depending on the severity of the disease. Mild disease might concern more frequently young patients who usually have otolaryngological symptoms including $\mathrm{OD}, \mathrm{GD}$ and $\mathrm{AD}$, while elderly patients have a higher risk to develop moderate-to-critical COVID-19, which are both characterized by general symptoms (e.g., cough, dyspnea, anorexia, nausea) and fewer otolaryngological disorders. In other words, the development of OD, GD and, to a lesser extend, AD may be considered as good prognostic outcomes of COVID-19 because less associated with moderate and severe-to-critical forms of the disease. Similar findings have been suggested in a recent cohort study of 949 patients who did not benefit from psychophysical evaluations [15].

The association between mild COVID-19 form and the development of OD is not yet elucidated. We suspect that the spread of severe acute respiratory syndrome coronavirus-2 (SARS-CoV-2) into the olfactory neuroepithelium and the olfactory bulb may lead to the development of local immunological reaction, which limits the spread of the virus in the host but leads to injuries of the neuroepithelium and olfactory bulb. This neurological hypothesis is supported by recent studies that identified injuries and edema of the olfactory cleft [16] and abnormalities of olfactory bulb on the magnetic resonance imaging [17]. Moreover, SARS-CoV-2 was identified in brain and olfactory bulb of non-survivors in a cadaveric study [18]. According to our data and the literature, the presence of otolaryngological symptoms and related OD could be a sign of a local inflammatory reaction, which could limit the virus spread into the host. The lack of association between SNOT-22 items and total score and the development of OD support the neurological process and not the OD related to nasal obstruction, which is frequently observed in usual viral infections such as common cold. Patients with subjective OD would have higher proportions of GD and AD. Although we used NHNES questionnaire, which is validated, it is possible that many patients with isolated OD had 'flavor disorder' and, therefore, confused $\mathrm{OD}, \mathrm{GD}$ and AD. For this reason, it is important to conduct future studies using psychophysical olfactory assessments and gustometry, so as to establish the potential association between these sense disorders. About the positive association between subjective and psychophysical olfactory evaluations, our results supported those of previous study [19].

In addition to the age and the disease severity, many conditions were found to be associated with the development of $\mathrm{OD}, \mathrm{GD}$ or $\mathrm{AD}$. In our study, diabetes was associated with the development of GD and there was a trend of association with Sniffin'Stick test results. The association between diabetes and the development of OD is well known and supported by a recent meta-analysis [20]. Similar findings were observed in patients with GD, confirming the potential role of diabetes in the development of sense disorders [21]. In this study, we found that patients with hypertension had a lower proportion of subjective $\mathrm{OD}$ and $\mathrm{AD}$ compared with those without hypertension. The role of hypertension and the potential intake of angiotensin converting enzyme inhibitors in the development of $\mathrm{OD}, \mathrm{GD}$ and $\mathrm{AD}$ was not extensively studied because only one report argued that COVID-19 patients with hypertension could have a significantly lower risk of OD compared with those without hypertension [22]. In the same vein, the association between tobacco, COVID19 and $\mathrm{AD}$ is still unclear and requires future investigations using objective measurements or psychophysical evaluations of sense functions.

The occurrence of gender-related differences in COVID19 presentation and clinical course has already been suspected in recent investigations and confirmed in the present study [23]. Due to chromosomal, hormonal and inflammatory differences, females could have a better prognosis of the disease, a higher proportion of mild and moderate forms, and, therefore, a higher proportion of OD [10, 23, 24]. Moreover, we observed higher proportions of OD, GD, cacosmia and $\mathrm{AD}$ in females compared with males, supporting the existence of gender differences in the host response to the viral infection. This hypothesis has to be confirmed in future studies using objective methods of olfactory and gustatory evaluations.

The main strength of this study is the high number of patients and the use of standardized patient-reported outcome questionnaire. Moreover, to the best of our knowledge, this study is the first investigation that reported subjective and psychophysical OD according to the WHO clinical state of COVID-19 patients. Through our collected data, we have identified clinical outcomes associated with the development of subjective or psychophysical OD, GD or AD. The relevance 
of some of these factors was confirmed in a subset of patients benefiting from psychophysical olfactory evaluations.

The main weakness of the study is the delay between the onset of the OD and the subjective and psychophysical evaluations. However, in practice, it was difficult to perform olfactory evaluations at the onset of the OD because the health situation limited us in the realization of our evaluations in mild patients, who were home-managed according to European government decisions, and moderate-to-critical (hospitalized) patients who required intensive cares. In addition, we were unable to perform olfactory cleft examination through nasal fibroscopy regarding the health recommendations of the majority of European hospitals. The delayed inclusion of severe-to-critical survivors in the study and the lack of inclusion of dead patients who early died are another potential inclusion bias which did not allow us to draw a clear conclusion about the prevalence of OD, GD and AD in severe-to-critical COVID-19 patients.

\section{Conclusion}

The prevalence of subjective and psychophysical OD was higher in mild COVID-19 patients compared with moderate and severe-to-critical patients. Subjective GD and AD could be more prevalent in mild COVID-19 forms but future studies using objective taste measurements are needed to confirm these findings. Age, gender and diabetes were identified as favoring factors of development of $\mathrm{OD}, \mathrm{GD}$ or $\mathrm{AD}$; while hypertension was associated with a lower probability to develop OD.

\section{Funding FRMH Funding.}

\section{Compliance with ethical standards}

Conflict of interest The authors declare that they have no conflict of interest.

Ethical approval All procedures performed in studies involving human participants were in accordance with the ethical standards of the institutional and/or national research committee and with the 1964 Helsinki declaration and its later amendments or comparable ethical standards.

Research involving human participants and/or animals IRB approved the study protocol (CHUB2020-12).

Informed consent Electronic informed consent was obtained from all individual participants.

\section{References}

1. WHO website. www.who.int/docs Consulted July 24, 2020.

2. Lechien JR, Chiesa-Estomba CM, De Siati DR et al (2020) Olfactory and gustatory dysfunctions as a clinical presentation of mild-to-moderate forms of the coronavirus disease (COVID-19): a multicenter European study. Eur Arch Otorhinolaryngol. https ://doi.org/10.1007/s00405-020-05965-1

3. Lechien JR, Chiesa-Estomba CM, Hans S, Barillari MR, Jouffe L, Saussez S (2020) Loss of smell and taste in 2013 European mildto-moderation COVID-19 patients. Ann Int Med 173(8):672-675. https://doi.org/10.7326/M20-2428

4. Vaira LA, Hopkins C, Salzano G et al (2020) Olfactory and gustatory function impairment in COVID-19 patients: Italian objective multicenter-study. Head Neck. https://doi.org/10.1002/hed.26269

5. Hopkins C, Surda P, Kumar N (2020) Presentation of new onset anosmia during the COVID-19 pandemic. Rhinology 58(3):295298. https://doi.org/10.4193/Rhin20.116

6. World Health Organization, 2020. Clinical Management of COVID-19: interim guidance, 27 may 2020 (No. WHO/2019nCoV/clinical/2020.5). World Health Organization.

7. Bhattacharyya N, Kepnes LJ (2015) Contemporary assessment of the prevalence of smell and taste problems in adults. Laryngoscope 125:1102-1106. https://doi.org/10.1002/lary.24999

8. Hummel T, Kobal G, Gudziol H et al (2007) Normative data for the "Sniffin' Sticks" including tests of odor identification, odor discrimination, and olfactory thresholds: an upgrade based on a group of more than 3,000 subjects. Eur Arch Otorhinolaryngol 264:237-243. https://doi.org/10.1007/s00405-006-0173-0

9. de Dorlodot C, Horoi M, Lefebvre P, Collet S, Bertrand B, Eloy P, Poirrier AL (2015) French adaptation and validation of the sino-nasal outcome test-22: a prospective cohort study on quality of life among 422 subjects. Clin Otolaryngol 40(1):29-35. https ://doi.org/10.1111/coa.12315

10. Lechien JR, Chiesa-Estomba CM, Place S, Van Laethem Y, Cabaraux P, Mat Q, Huet K, Plzak J, Horoi M, Hans S, Rosaria Barillari M, Cammaroto G, Fakhry N, Martiny D, Ayad T, Jouffe L, Hopkins C, Saussez S, COVID-19 Task Force of YO-IFOS (2020) Clinical and epidemiological characteristics of 1420 European patients with mild-to-moderate coronavirus disease 2019. J Intern Med. https://doi.org/10.1111/joim.13089

11. Moein ST, Hashemian SMR, Mansourafshar B, Khorram-Tousi A, Tabarsi P, Doty RL (2020) Smell dysfunction: a biomarker for COVID-19. Int Forum Allergy Rhinol. https://doi.org/10.1002/ alr.22587

12. Chiesa-Estomba CM, Lechien JR, Portillo-Mazal P, Martínez F, Cuauro-Sanchez J, Calvo-Henriquez C, Saussez S (2020) Olfactory and gustatory dysfunctions in COVID-19. First reports of Latin-American ethnic patients. Am J Otolaryngol 41(5): 102605. https://doi.org/10.1016/j.amjoto.2020.102605

13. Lee Y, Min P, Lee S, Kim SW (2020) Prevalence and duration of acute loss of smell or taste in COVID-19 patients. J Korean Med Sci 35(18):e174. https://doi.org/10.3346/jkms.2020.35.e174

14. Speth MM, Singer-Cornelius T, Obere M, Gengler I, Brockmeier SJ, Sedaghat AR (2020) Olfactory dysfunction and sinonasal symptomatology in COVID-18: prevalence, severity, timing, and associated characteristics. Otolaryngol Head Neck Surg. https:// doi.org/10.1177/0194599820929185

15. Foster KJ et al (2020) Smell loss is a prognostic factor for lower severity of COVID-19. Ann Allergy Asthma Immunol 125(4):481-483. https://doi.org/10.1016/j.anai.2020.07.023

16. Lechien JR, Michel J, Radulesco T, Chiesa-Estomba CM, Vaira LA, De Riu G, Sowerby L, Hopkins C, Saussez S (2020) Clinical and radiological evaluations of COVID-19 patients with anosmia: preliminary report. Laryngoscope. https://doi.org/10.1002/ lary. 28993

17. Chetrit A, Lechien JR, Ammar A, Chekkoury-Idrissi Y, Distinguin L, Circiu M, Saussez S, Ballester MC, Vasse M, Berradja N, Hans S, Carlier R, Edjlali M (2020) Magnetic resonance imaging of COVID-19 anosmic patients reveals abnormalities of the olfactory 
bulb: preliminary prospective study. J Infect 81(5):816-846. https ://doi.org/10.1016/j.jinf.2020.07.028

18. Coolen T, Lolli V, Sadeghi N, Rovaï A, Trotta N, Taccone FS, Creteur J, Henrard S, Goffard JC, De Witte O, Naeije G, Goldman S, De Tiège X (2020) Early postmortem brain MRI findings in COVID-19 non-survivors. Neurology. https://doi.org/10.1212/ WNL.0000000000010116

19. Liu DT, Besser G, Prem B, Sharma G, Koenighofer M, Renner B, Mueller CA (2020) Association between orthonasal olfaction and chemosensory perception in patients with smell loss. Laryngoscope 130(9):2213-2219. https://doi.org/10.1002/lary.28773

20. Kim SJ, Windon MJ, Lin SY (2019) The association between diabetes and olfactory impairment in adults: a systematic review and meta-analysis. Laryngoscope Investig Otolaryngol 4(5):465-475. https://doi.org/10.1002/lio2.291

21. Hu C, Yao L, Chen Z, Sun Z, Zhang L, Wei Y (2020) Assessment of gustatory function in aging people using event-related potentials. NeuroReport 31(9):650-656. https://doi.org/10.1097/ WNR.0000000000001457

22. Lechien JR, Barillari MR, Jouffe L, Saussez S (2020) Anosmia is a key symptom of COVID-19 infection and should be used as a diagnostic tool. Ear Nose Throat J. https://doi.org/10.1177/01455 61320925191

23. Chanana N, Palmo T, Sharma K, Kumar R, Graham BB, Pasha Q (2020) Sex-derived attributes contributing to SARS-CoV-2 mortality. Am J Physiol Endocrinol Metab. https://doi.org/10.1152/ ajpendo.00295.2020

Publisher's Note Springer Nature remains neutral with regard to jurisdictional claims in published maps and institutional affiliations.

\section{Authors and Affiliations}

\section{Jerome R. Lechien ${ }^{1,2,3,4}$ (C) Carlos M. Chiesa-Estomba ${ }^{1,5}$ - Luigi A. Vaira ${ }^{6}$ - Giacomo De Riu ${ }^{6}$. Giovanni Cammaroto ${ }^{1,7}$. Younes Chekkoury-Idrissi ${ }^{3}$. Marta Circiu ${ }^{3}$. Lea Distinguin ${ }^{3}$. Fabrice Journe ${ }^{2}$. Christophe de Terwangne ${ }^{8}$. Shahram Machayekhi ${ }^{8} \cdot$ Maria R. Barillari $^{1,9} \cdot$ Christian Calvo-Henriquez $^{10} \cdot$ Stéphane Hans $^{3} \cdot$ Sven Saussez $^{1,2,4}$}

1 COVID-19 Task Force of the Young-Otolaryngologists of the International Federations of Oto-rhino-laryngological Societies (YO-IFOS), Paris, France

2 Department of Human Anatomy and Experimental Oncology, Faculty of Medicine, UMONS Research Institute for Health Sciences and Technology, University of Mons (UMons), Mons, Belgium

3 Department of Otolaryngology-Head and Neck Surgery, Foch Hospital, School of Medicine, UFR Simone Veil, Université Versailles Saint-Quentin-en-Yvelines (Paris Saclay University), Paris, France

4 Department of Otorhinolaryngology and Head and Neck Surgery, CHU de Bruxelles, CHU Saint-Pierre, School of Medicine, Université Libre de Bruxelles, Brussels, Belgium
5 Department of Otorhinolaryngology-Head and Neck Surgery, Hospital Universitario Donostia/ Biodonostia Research Institute, San Sebastian, Spain

6 Maxillofacial Surgery Unit, University Hospital of Sassari, Sassari, Italy

7 Department of Otolaryngology-Head and Neck Surgery, Morgagni Pierantoni Hospital, Forli, Italy

8 Intensive Care Unit, EpiCURA Hospital, Hornu, Belgium

9 Division of Phoniatrics and Audiology, Department of Mental and Physical Health and Preventive Medicine, University of Naples SUN, Naples, Italy

10 Department of Otorhinolaryngology and Head and Neck Surgery, Hospital Complex of Santiago de Compostela, Santiago de Compostela, Spain 